\title{
The Reemergence of Specialty Pharmacy
}

\author{
Gannon Vanscoy, Brandeis Seymore, RPh; Nivedita Kohli, RPh; \\ Jigneshkumar Patel, RPh; and Jonathan Ogurchak, PharmD, CSP
}

\begin{abstract}
SUMMARY
Twenty years ago, the Journal of Managed Care \& Specialty Pharmacy published an article titled "The Emergence of Specialty Pharmacy." While the industry was in its relative infancy at the time, the specialty pharmacy model has since grown, expanded, and matured, largely following some of the trends outlined at the time. Now, with changes in legislation, a progressive approach within the FDA, a second coming of novel therapies and supplemental indications, along with an involvement in cell and gene therapy, a reemergence of the specialty model is taking place, and the market must adapt to the new challenges associated with this era of modern medicine.
\end{abstract}

J Manag Care Spec Pharm. 2020;26(3):234-35

Copyright $\odot 2020$, Academy of Managed Care Pharmacy. All rights reserved.

$\mathrm{T}$ he U.S. drug pipeline is swelling with new and transformative therapies. Nearly 7,000 medicines are currently in development, with almost $75 \%$ of them potentially first in class). ${ }^{1}$ The U.S. Food and Drug Administration (FDA) continues to set records for novel therapy approvals, and in 2018, the Center for Drug Evaluation and Research (CDER) reported that there were 59 novel therapies and biologics approved. Of these, more than half were for rare or orphan diseases; 14 were designated breakthrough therapies; and 16 were to treat different cancers. ${ }^{2}$ Furthermore, drugs may receive priority review if CDER determines that the drug could potentially provide a significant advance in medical care, helping to expedite the time from clinic to commercialization.

\section{Rare and Orphan Focus}

One reason for this tremendous growth is a reinvigorated focus on drug development in rare and orphan disease states. Currently, only $7 \%$ of rare diseases have treatments. ${ }^{3}$ There are more than 450 therapies in later stage development to treat these rare and devastating diseases. This is bolstered by favorable legislation supporting development through extended market exclusivity, tax incentives, and the pediatric voucher program. ${ }^{3}$ In the last 5 years, there has been a consistent growth in the percentage of FDA approvals targeted towards orphan-designated diseases. ${ }^{4}$ Many of these patient populations previously had no effective treatment options.

Orphan drugs bring with them unique challenges in the patient journey, including a need for clinical services, financial accessibility, and distribution modality to patient. Specialty pharmacy involvement has expanded to include nursing programs, case management, enhanced reimbursement, 24/7 clinical support, patient-assistance program qualification, and quick start programs in order to support the needs of a limited distribution therapy. Most commercial launches for orphan drug products have shifted to highly limited (2-4 specialty pharmacies) or exclusive (1 specialty pharmacy) distribution networks with the intention of containing costs in the supply chain and providing an enhanced and consistent experience to patients not seen as frequently at the pharmacy.

As a result, specialty pharmacies have also responded to market a focus on orphan drugs, as opposed to the general specialty therapies seen since the year 2000. Third-party accreditors, a growing staple in specialty pharmacy validation since the mid-2000s, have expanded new standards to focus on the needs of these therapies. In the fall of 2019, the Accreditation Commission for Health Care released standards for an accredited distinction in rare disease management. URAC is also currently developing a rare pharmacy designation, which is expected to launch in 2020

\section{Taking a More Personalized Approach}

Of the estimated 7,000 identified rare diseases, $80 \%$ of these conditions are estimated to be caused by a faulty gene. ${ }^{5}$ Transformative gene and gene therapies are now being researched, where vectors (in the form of drugs) are placed in the patient to replace the damaged gene. Currently, there are over 900 cell and gene therapy clinical trials in progress. ${ }^{6}$ Many of these therapies present logistical and financial challenges, such as requiring advanced cold-chain storage and shipment at cryogenic temperatures below -60 or -150 degrees Celsius. Timing is also a critical element for certain "one-shot" treatments, with a limited time frame of efficacy once the therapies are prepared.

Current regulations require a pharmacy to physically touch and inspect the product before dispensing. With a drug that has been developed specifically for a patient, which then needs to be transported to the location of administration, the channel execution and logistical coordination must be flawless. 


\section{Challenges and Opportunities}

Marketplace realities pose new challenges for patients, employers, insurers, and specialty pharmacies, particularly with maintaining patient access while keeping drug spend in check. Along with growing costs, increase in incidence of complex conditions and fragmented care present additional challenges. Formulary management provides lowest net cost options for members and payors and includes clinically appropriate, costeffective drug options. Applying evidence-based guidelines through prior authorization (PA) ensures that the right patient has the right drug at the right time. PA through technology platforms provides greater control of diagnosis review, clinical data, and response to therapy as a condition of coverage. Pharmacy benefit managers help to encourage the use of preferred, lowest-cost drug products for additional savings and optimize the site of care where certain specialty products can be administered, such as in a patient's home instead of in more expensive outpatient facilities or physician offices.

At the same time, the need to better manage and support patients with specialty conditions is critical. This goal is often hindered by the lack of connected care and alignment among key stakeholders across the health care system. Today, each stakeholder must try to make appropriate decisions with imperfect and incomplete information. Disconnected and disjointed systems, many of them manual, make it difficult to select the most appropriate medication therapy, track ongoing adherence, counsel patients about needed interventions, and ensure that patients are fully informed about the most effective drug options.

As consumerism continues to increase, patients have certain needs and expectations when it comes to accessing information needed to make informed decisions about their health. The need for effective patient engagement has become even more critical. While traditional nurse support and disease management interventions are critical for specialty pharmacies, it is equally important to engage patients in more fully understanding costs of care, providing tools to help more efficiently navigate benefits and out-of-pocket expenditures.

One method for improving patient engagement is through partnering with specialty pharmacy hubs when launching products with a limited distribution network. This method can reduce confusion, providing a consistent branded solution to patient engagement. Hubs are able to efficiently and appropriately distribute referrals to the specialty pharmacy in a manufacturer's network; provide enhanced services such as nursing support, case management, adherence programs, reimbursement support, and patient-assistance program qualification; and ensure a consistent patient experience no matter which dispensing specialty pharmacy is used.

\section{The Next 20 Years}

As an industry, specialty pharmacy has come a long way since the year 2000, when it was highlighted by JMCP. With the majority of new drug development targeting potential specialty therapies, the future is bright for the next 20 years. Advancements in novel therapies, along with the challenges of affordability, will continue to push improvements in patient care to ensure the best overall patient experience.

\section{Authors}

GANNON VANSCOY; BRANDEIS SEYMORE, RPh; NIVEDITA KOHLI, RPh; and JIGNESHKUMAR PATEL, RPh, Master of Pharmacy Business Administration candidates, University of Pittsburgh, Pittsburgh, Pennsylvania. JONATHAN OGURCHAK, PharmD, CSP, Adjunct Faculty, University of Pittsburgh, and CEO, STACK for Pharmacy, Pittsburgh, Pennsylvania.

AUTHOR CORRESPONDENCE: Jonathan Ogurchak, PharmD, CSP, Adjunct Instructor, MPBA Program, University of Pittsburgh, 3501 Terrace St., Pittsburgh, PA 15213.E-mail: JMO63@pitt.edu.

\section{DISCLOSURES}

No funding contributed to the writing of this article. Ogurchak reports speaker fees from MJH Live Events and Wellsky, unrelated to this work. The other authors have nothing to disclose with respect to research, authorship, and/or publication of this article.

\section{REFERENCES}

1. Pharmaceutical Research and Manufacturers of America. In the pipeline: what's next in drug discovery. Available at: https://www.phrma.org/science/ in-the-pipeline. Accessed January 27, 2020.

2. U.S. Food and Drug Administration, Center for Drug Evaluation and Research. Novel drug approvals for 2018. Available at: https://www.fda.gov/ drugs/new-drugs-fda-cders-new-molecular-entities-and-new-therapeuticbiological-products/novel-drug-approvals-2018. Accessed January 27, 2020.

3. Orphanet. About orphan drugs. Available at: https://www.orpha.net/ consor/cgi-bin/Education_AboutOrphanDrugs.php?lng=EN\&stapage=ST_ EDUCATION_EDUCATION_ABOUTORPHANDRUGS_USA\#providers. Accessed January 27, 2020.

4. Brennan, Z. FDA: 2019 continues uptick in orphan drug approvals. Regulatory Focus. January 6, 2020. Available at: https://www.raps.org/newsand-articles/news-articles/2020/1/fda-2019-continues-uptick-in-orphandrug-approval. Accessed January 27, 2020.

5. National Institutes of Health, Genetic and Rare Diseases Information Center. FAQs about rare diseases. Available at: https://rarediseases.info.nih gov/diseases/pages/31/faqs-about-rare-diseases. Accessed January 27, 2020.

6. Alliance for Regenerative Medicine. Annual data report, 2017. Available at: https://alliancerm.org/wp-content/uploads/2018/05/ARM_Annual_ Report_2017_FINAL.pdf. Accessed January 27, 2020. 